\title{
Diagnosis and Treatment Modalities for Temporomandibular Disorders (Part I): History, Classification, Anatomy and Patient Evaluation
}

\author{
${ }^{1}$ Satheesh Simha Reddy Panga, ${ }^{2}$ Ravi Sekhar, ${ }^{3}$ Raja Sekhar \\ ${ }^{1}$ Assistant Professor, Department of Prosthodontics, Army College of Dental Sciences, Secunderabad, Andhra Pradesh, India \\ ${ }^{2}$ Professor, Department of Maxillofacial Surgery, Navodaya Dental College, Raichur, Karnataka, India \\ ${ }^{3}$ Professor and Head, Department of Oral Surgery, Mamata Dental College, Khammam, Andhra Pradesh, India
}

Correspondence: Satheesh Simha Reddy Panga, Consultant Prosthodontist, Vivekananda Dental Clinic, Vivekananda Hospital Hyderabad, Andhra Pradesh, India, Phone: +919966225888, e-mail: satish.mds@gmail.com

\section{ABSTRACT}

Temporomandibular disorder (TMD) is not just one disorder, but a group of conditions, often painful, that affect the temporomandibular joint, and the muscles that control chewing. Many psychological and physical factors cause myofascial pain, internal derangement of the joint, degenerative joint diseases which ultimately leads to temporomandibular disorder. Scientists are exploring how behavioral, psychological and physical factors may combine to cause temporomandibular disorders and researchers are working to clarify temporomandibular disorders symptoms, with the goal of developing easier and better methods of diagnosis and improved treatment. In part 1 of this 2-part series on temporomandibular disorders, emphasis will be placed on the history and classification of temporomandibular disorders, structure and function of the temporomandibular joint (TMJ), and patient evaluation techniques.

Keywords: Temporomandibular disorders, Orofacial pain, Temporomandibular joint.

\section{INTRODUCTION}

Temporomandibular disorders (TMDs) include a number of clinical problems that involvethe masticatory musculature, the temporomandibular joint and associated structures. ${ }^{1}$ They are considered to be a subclassification of musculoskeletal disorders. Detailed history taking and standardized examination techniques are crucial informing a proper differential diagnosis. The patient may present with jawache, earache, toothache, facial pain, and/or headache; however, the chief complaint may be as a general facial tightness or fatigue. Treatment planning is dependent on various factors such as the chief complaint, health history, presenting symptoms, examination and diagnosis. In the past, TMD cases have sometimes been regarded as problematic to diagnose and difficult to treat; however, with continuing research in orofacial pain and pain management, clinicians are able to use specific diagnostic methods and standardized classification systems to offer patients treatment modalities with higher success rates.

\section{HISTORY AND EVOLUTION OF THE MANAGEMENT OF TMD (TABLE 1)}

As a result of Costen's work, awareness of the TMJ and its relationship to facial pain increased greatly among practitioners. ${ }^{2} \mathrm{H}$ e noted that a number of his patients with pain in the TMJ region seemed to experience a reduction of symptoms following therapeutic alteration in their occlusion. These findings were significant on two fronts. First, Costen's writings introduced hypotheses that occlusion and TM D may be linked. Second, he emphasized the practitioner's role in providing treatments aimed at affecting the TMJ region. Thus, in the first half of the century, generally accepted concepts of patient management focused on occlusal adjustment as the major treatment modality for TM D. ${ }^{3-7}$

Eventually, concepts were proposed that challenged occlusal ly-based treatments. ${ }^{8,9}$ In 1969, Laskin ${ }^{10}$ postulated a theory emphasizing the role of chronic oral habits brought on by emotional stress and its effect on muscle spasm, pain and fatigue. Hence, clinicians began considering stress as one of the prime etiological contributors to TMD and the term myofascial pain dysfunction syndrome emerged. In the 1970s, detailed focus on the anatomical structure of the TMJ led to increased understanding of TMD etiology. Wilkes, ${ }^{11}$ Farrar, and $\mathrm{M} \mathrm{CC}$ arthy ${ }^{12,13}$ were among those who studied the diskcondylar complex. During this time, Solberg and Rugh ${ }^{14}$ continued to emphasize the role played by psychological factors and stress on the musculature.

The concept of internal derangement of the TMJ was introduced by Ireland ${ }^{15}$ in 1953 but was not widely accepted until the 1970s, following imaging techniques, such as arthrography. Also in the late 1970s and 1980s, surgical intervention with disk repositioning started gaining popularity. Subsequently, TMJ disk removal procedures were recommended, with some surgeons choosing to maintain the joint complex without a disk while others opted for prosthetic TM J disk implant placement. Unfortunately, use of certain 
Diagnosis and Treatment Modalities for Temporomandibular Disorders (Part I): History, Classification, Anatomy

Table 1: History of the clinical management of temporomandibular disorders

\begin{tabular}{lll}
\hline & \multicolumn{2}{c}{ Table 1: History of the clinical management of temporomandibular disorders } \\
\hline Years & Investigators & Concepts \\
\hline $1920-30 s$ & McCollum, Stallard and Stuart & Gnathological concepts \\
1934 & Costen & Overclosure/vertical dimension of occlusion was the primary cause of TM D \\
1950 s & Shore & Occlusal equilibration \\
1951 & Ireland & M echanisms of TMJ clicking \\
1952 & Travell and Rizzler & M yofascial genesis of pain \\
1956 & Schwartz & E motional tension as a contributing factor \\
1961 & Ramfjord & Occlusal equilibration \\
1969 & Laskin & Pain dysfunction syndrome secondary to oral habits and muscle pain \\
1970 & Ohnishi & A rthroscopy in the TM J \\
1971 & Farrar & Internal derangement \\
1974 & Greene and Laskin & M yofascial pain dysfunction syndrome \\
1976 & Rugh and Solberg & Stress as an etiological factor \\
1978 & Wilkes & TMJ arthrography \\
1983 & Scapino & Pseudodisk formation \\
1986 & Sanders & A rthroscopic surgery of TM J \\
1991 & Nitzan, Dolwick and M artinez & A rthrocentesis \\
2000 & A merican A cademy for Orofacial Pain & Comprehensive orofacial pain management \\
\hline
\end{tabular}

prosthetic implants, such as V itek proplast/teflon resulted in severe complications, such as giant cell foreign body reactions. In some implant cases, this led to extremely painful joint conditions with limited range of motion (ROM ). L ess-invasive surgical techniques, such as arthrocentesis and arthroscopy proved to be highly effective for chronic TM J conditions (e.g. unresolving joint pain and closed lock). ${ }^{16-18}$

Today, there is a greater knowledge of the complex mechanisms involved in chronic pain conditions as well as a greater appreciation of the role played by the affective and cognitive components of pain. Together with increased emphasis on evidence-based treatments, practitioners are using less-invasive treatment modalities. Clinicians, having learned to equate TMD with other chronic pain conditions, can now manage TM D in a comprehensive and scientific manner.

\section{STRUCTURE AND FUNCTION}

TM J has been a source of interest since the fifteenth century, when this anatomical structure was studied by Leonardo da Vinci. Other notable contributors include Vesalius in the sixteenth century, M eyers in the nineteenth century, and Sicher ${ }^{19}$ and Rees ${ }^{20}$ in the early twentieth century. The TM J has been described as a ginglymoarthrodial synovial compound paired joint. A compound joint consists of three bones; however, in the case of the TM J, the functional disk operates as the third nonossified bone and therefore fulfills the criteria (Fig. 1). A s a synovial joint, it is governed by the same basic orthopedic principles that apply to other human synovial joints, ranging from pathologic disorders to treatment protocols. The $\mathrm{TM}$ J, however, has many unique features that distinguish it from other synovial joints. These include (1) rigid end point of closure; (2) one side cannot function without movement of the opposite joint; (3) both joints act as one functional unit; (4) any movement or functional al teration in one joint will affect the other joint; and (5) no hyaline cartilage is present.

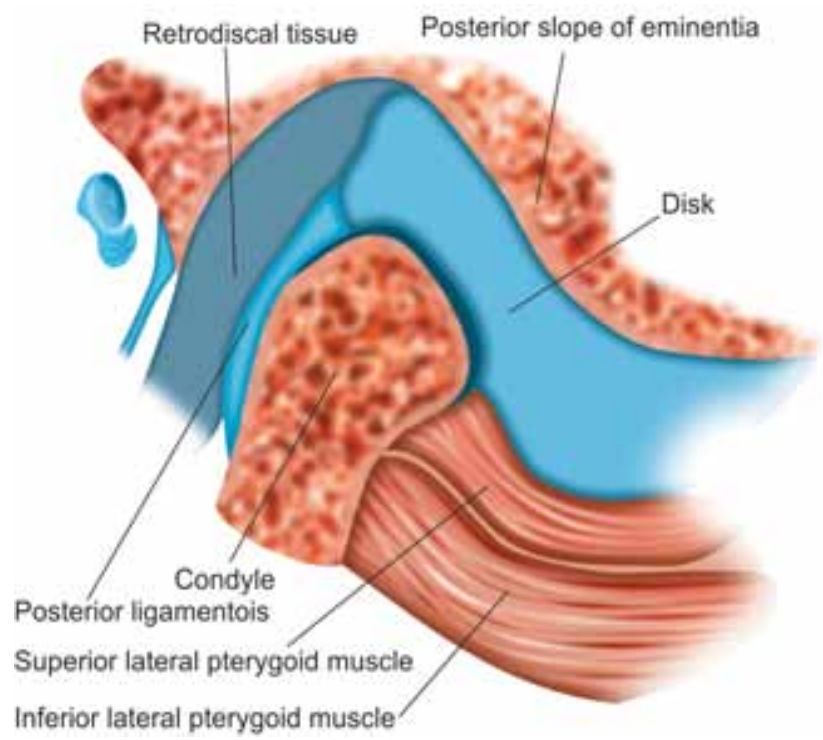

Fig. 1: Structure of the temporomandibular joint

The TM J articulates between the condyle of the mandible and the glenoid fossa of the temporal bone, situated between the bony components is the articular disk. It consists of dense fibrous connective tissue and is devoid of nerves and blood vessels in the articulating portion; however, the posterior attachment has rich vascularization and innervation via the auriculotemporal branch of the mandibular division of the trigeminal nerve. It is biconcave in shape, with the thickest portion in the posterior zone, a moderately thick anterior zone, and a thin intermediate zone. The joint capsule is attached to the disk and divides the joint into two compartments. The associated ligaments include the functional ligaments (collateral, capsular and temporomandibular) and the accessory ligaments (sphenomandibular and stylomandibular). The purpose of the ligaments is to protect the joint by restricting and limiting border movements. The associated muscles of mastication include the masseter, temporalis, medial and lateral 
pterygoid and the digastric. A clear understanding of the anatomical components and the function of the TMJ is crucial in forming a proper differential diagnosis and ultimately developing an individualized treatment plan.

\section{HISTORY}

In a study on symptoms associated with TM D, Rasmussen ${ }^{21}$ found that most patients with a clicking TMJ usually did not evolve into an open or closed locking state. According to his findings, acute TM D symptoms lasted a mean of 5 years, and al though joint noises generally did not disappear, most painful and disabling symptoms subsided in time. Similar results were shown by Könönen et al, who followed 128 Finnish adults over 9 years. ${ }^{22}$ They found that the incidence of clicking in these patients increased with age. However, none of the patients developed locking. These studies demonstrate the importance of reversible and noninvasive treatments for the acute TM D patient. In an epidemiological study by Solberg, ${ }^{23} 76 \%$ of subjects aged 18 to 25 had one or more signs associated with TM D and $26 \%$ had at least one symptom associated with TM D. Of this group, only $10 \%$ had symptoms that were considered by the subjects to be severe enough to seek treatment.

M ost epidemiological studies clearly demonstrate that TM D symptoms are more commonly seen in women than in men. ${ }^{1}$ Symptoms often arise from adolescence to early twenties and may continue intermittently well into middle age. Multiple etiological factors have been associated with TM D (Table 2).

Table 2: Possible etiological factors in the development of temporomandibular disorders

- Stress

- External trauma

- Bruxism

- Systemic poly joint arthritis

- Hypermobility disorder

- Neoplasia

- Developmental/growth abnormality

- Dental morphologic abnormality

- Daytime habitual behavior

- Infection

- Idiopathic

\section{CLASSIFICATION (TABLES 3 AND 4)}

\section{Internal Derangement (ID)}

Internal derangement of the TMJ is characterized by an anatomical disturbance in the relationship of the components of the disk-condyle complex. This term encompasses a number of clinical entities, including the following:

D isk-condyle incoordination: This term describes patients with a distinct joint noise, such as a click or pop, with deviation in the pattern of opening. Other terms used to describe this condition are (1) disk displacement with reduction and (2) anterior or medial disk displacement.
Table 3: Classification of temporomandibular disorders

1. Articular

- Internal derangement

- Inflammatory joint disorders

2. Muscular

- Myalgia

- Myositis

- Myofascial pain

Table 4: Inflammatory joint disorders osteoarthrosis

Osteoarthritis

Polyarthritis

Traumatic

Infectious

Rheumatoid

Hyperuricemia

Psoriatic

A nkylosing spondylitis

Disk-condyle restrictions: This usually occurs in a patient with a history of clicking. On examination, the patient reports a sudden onset in hypomobility, usually measurable as an opening of less than $30 \mathrm{~mm}$, and a cessation of joint noises. The pati ent deflects to the locked side on opening, often with pain. In a majority of cases, the disk is anteriorly displaced, while posterior disk displacement happens rarely. A Iternative terms include disk displacement without reduction and closed locking.

0 pen locking: In this scenario, the condyle is anterior to the eminence and the patient is locked in a wide-open position. The patient may be able to self-reduce without any assistance.

Condyle dislocation: This differs from open locking in that the condyle has undergone excessive translation well beyond the eminence and requires manual manipulation to return to a normal position. A TMJ tomogram or M RI can help confirm this diagnosis.

Partial open lock: These patients complain of an inability to close their jaw after opening, but on radiographic examination, the condyle is not beyond the eminence. The disk may be dislocated in a posterior position, thus preventing the condyle from seating correctly.

\section{Inflammatory Joint Disorders}

Arthralgia: This describes a joint that displays increased tenderness on pal pation. Osseous changes are usually not noted on radiographic examination. $V$ arious terms have been used to specifically characterize this condition depending upon the location of the pain (e.g. capsulitis, retrodiscitis and synovitis).

Arthritides: A $n$ inflammatory process of the articular surface of a joint that may be localized to one joint or may be generalized, affecting multiple joints (polyarthritis). 


\section{Muscle Disorders}

M yalgia/myofascial pain (MFP): This is a dull, aching pain varying in intensity. The primary difference between M FP and myalgia is that M FP produces pain referred to other satellite muscle trigger points upon pal pation, whereas myalgia results in pain that is localized to the muscle that is being palpated. M FP tends to be seen in more chronic muscle pain conditions compared to the usually acute presentation of myalgia. Palpation of the trigger points should duplicate the patient's pain complaint, thus confirming the diagnosis. In addition, blocking the source of the pain (i.e. masseter muscle) utilizing a vapocoolant spray or local anesthetic injection can also provide a definitive diagnosis.

M yositis: This presents as a localized transient inflammation involving muscle tissues. Classically, there is increased pain with mandibular movement and localized tenderness, usually following injury or infection.

\section{Patient Evaluation}

Temporomandibular disorder assessment should include a general examination of the head and neck, a detailed examination of the masticatory muscles, an evaluation of the temporomandibular joints, an evaluation of mandibular range of motion and a detailed intraoral examination. ${ }^{24}$

\section{Evaluation of the Muscles of Mastication}

The muscles of mastication are pal pated bilaterally for firmness and tenderness utilizing approximately 2 to 3 pounds of pressure. A pain pressure al gometer may be used to reproduce reliable palpation pressure ${ }^{25}$ or the amount of pressure needed to cause blanching of the fingernail. U pon muscle palpation, the patient is asked to report the severity of the tenderness, pain referral to multiple sites or single-site pain localization and replication of the chief complaint. The primary muscles to be pal pated include the temporalis (Fig. 2), the superficial and deep masseter (Fig. 3), the medial and lateral pterygoid, the suprahyoid and the upper cervical muscles. N ote that pal pation of the lateral pterygoid from an intraoral perspective is difficult. ${ }^{26}$ It may be pertinent to ask patients about their use of analgesic prior to palpation in order to account for reduced symptoms upon examination.

\section{Evaluation of the Temporomandibular Joint}

The TM J s are pal pated bilaterally for tenderness or swelling with slightly less pressure than used for muscle palpation, especially in the presence of an existing capsulitis (Fig. 4). The clinician should palpate the preauricular region as well as the anterior walls of the external auditory canal. Lateral palpation may be utilized to assess joint pain in the lateral capsule, whereas the intrameatal approach via the external auditory canal is better for locating pain emanating from retrodiscal tissues. ${ }^{1}$

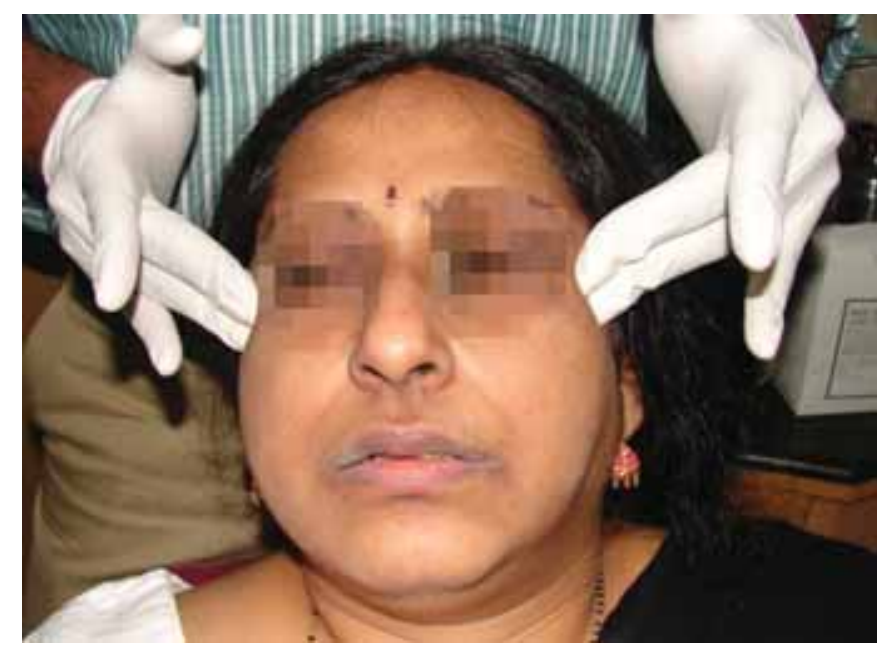

Fig. 2: Palpating the temporalis muscle

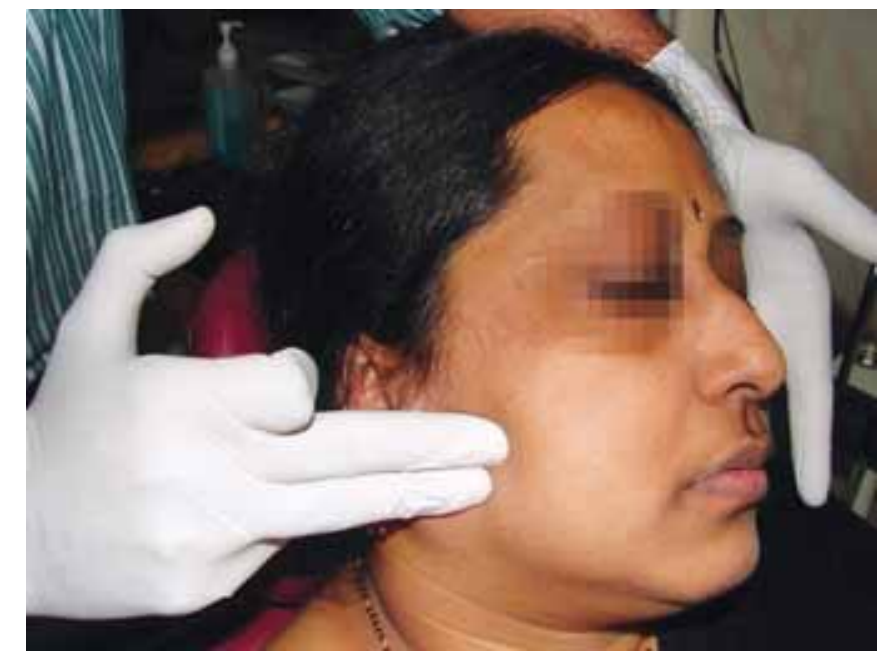

Fig. 3: Palpating the superficial and deep masseter muscles

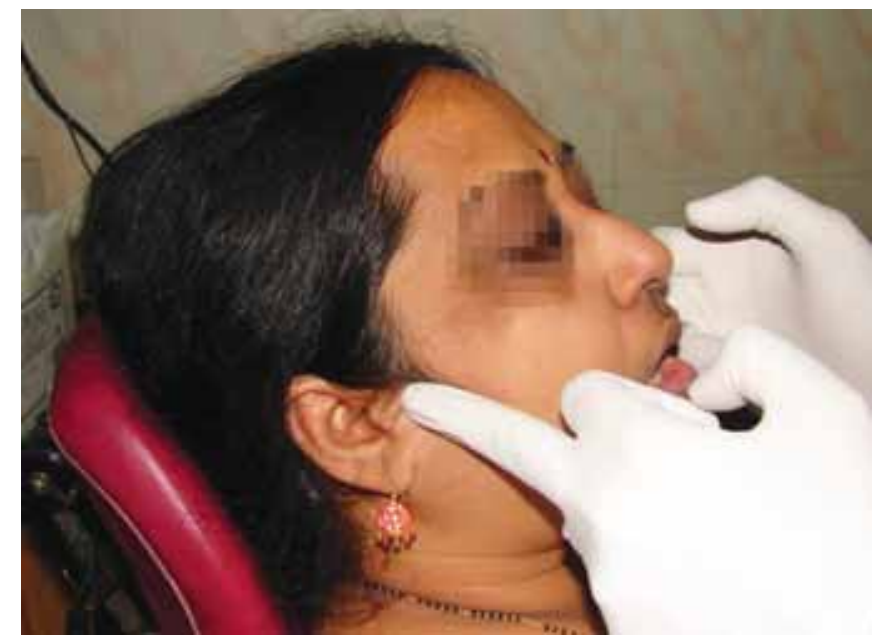

Fig. 4: Bilateral palpation of the TMJs

\section{Evaluation of Mandibular Range Motion (ROM)}

Initially, patients' opening and closing patterns are closely observed to note any mandibular deviations. Evaluation of mandibular ROM consists of measuring with a millimeter ruler the (1) comfort opening, (2) active opening, (3) passive 
opening, (4) protrusion and (5) left and right lateral excursions while noting the severity and location of pain with jaw movement (Fig. 5). This can be particularly helpful in differentiating between joint and muscle pain. Comfort opening is determined by (1) the patient opening as wide as possible without any pain, (2) active opening with the patient opening as wide as possible with pain and (3) passive opening with the clinician gently stretching the patient presumably past active opening while noting a soft or hard end feel. A reasonably normal interincisal distance is approximately $40 \mathrm{~mm}$ or the width of 3 of the patient's fingers as a crude measure (Fig. 6). U sually, with proper questioning, the patient will reliably reveal any recent limitations in $\mathrm{ROM}$. The occurrence of $\mathrm{TM}$ J clicking, crepitus, or jaw opening interferences with or without pain should also be noted at the initial examination. These baseline findings will aid in establishing the differential diagnosis and treatment options as well as providing a comparison for future change in TM D symptoms.

\section{Evaluation of the Cervical Spine}

Patients who present with TMD may have a coexisting pain complaint in the neck, shoulder or upper back region. Poor posture may lead to a forward head position, rounded shoulders,

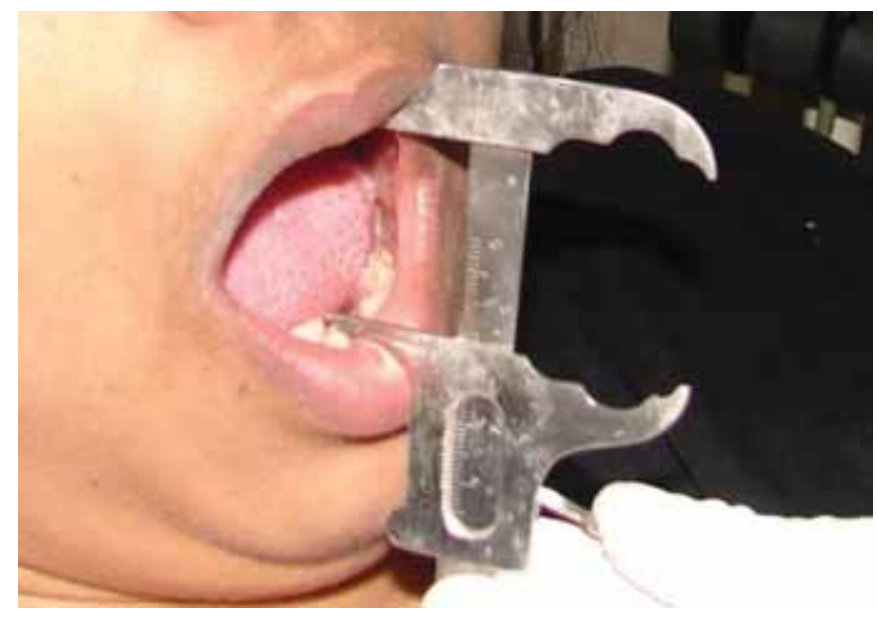

Fig. 5: Evaluating mandibular range of motion using a millimeter ruler

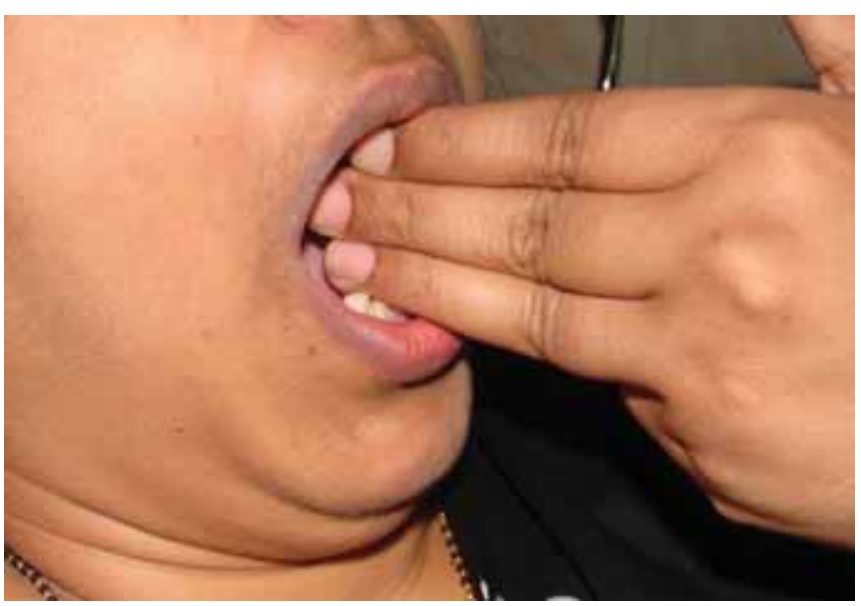

Fig. 6: A crude measure of reasonably normal interincisal distance is approximately the width of 3 of the patient's fingers and/or added tension in the head, neck or back. Thus, an evaluation of the cervical spine may aid in assessing the patient's head ROM in flexion, extension and rotation. In addition, if range of motion is limited, it is pertinent to note any areas of pain that the patient experiences while performing various head and neck movements. This may help in localizing additional trigger areas that may have been missed on the palpatory examination.

\section{SUMMARY}

The study of temporomandibular disorders has undergone many changes throughout its history. Focus on the structure and function of the TM J continues to improve our understanding of these complex disorders. A more standardized classification system allows practitioners and researchers to discuss findings in a common language. With improved patient evaluation techniques, the clinician can establish a proper working differential diagnosis and begin focusing attention on treatment planning.

\section{REFERENCES}

1. Okeson JP. O rofacial pain: Guidelines for assessment, diagnosis and management. Chicago, III: Quintessence Publishing; 1996.

2. Costen JB. A syndrome of ear and sinus symptoms dependent upon disturbed function of the temporomandibular joint. Ann Otol Rhinol Laryngol 1934;43:1-15.

3. M cCollum BB. Factors that make the mouth and teeth a vital organ (articulation orthodontia). J A m Dent Assoc 1927; 14:1261-71.

4. Stallard $\mathrm{H}$. Functions of the occlusal surfaces of the teeth. J A m Dent A ssoc 1930;13:401.

5. Stuart CE, Golden IB. The history of gnathology. V entura, Calif: CE Stuart G nathological Instruments 1981.

6. Shore N. Temporomandibular joint dysfunction and occlusal equilibration. Philadel phia, Pa: JB Lippincott 1976.

7. Ramfjord SP. Dysfunctional temporomandibular joint and muscle pain. J Prosthet Dent 1961;11:353-74.

8. Travell J, Rinzler SH. The myofascial genesis of pain. Postgrad M ed 1952;11:425-34.

9. Schwartz LL. A temporomandibular joint pain dysfunction syndrome. J Chronic Dis 1956;3:284-93.

10. Laskin DM . Etiology of the pain-dysfunction syndrome. J Am Dent A ssoc 1969;79:147-53.

11. Wilkes $\mathrm{CH}$. A rthrography of the temporomandibular joint in patients with the TMJ pain-dysfunction syndrome. M inn Med 1978;61:645-52.

12. Farrar WB. Diagnosis and treatment of anterior dislocation of the articular disk. NYJ Dent 1971;41:348-51.

13. Farrar W B, M CC arty WL. A clinical outline of temporomandibular joint diagnosis and treatment. M ontgomery, A la: N ormandie Study Group Publications, 1982.

14. Rugh JD, Solberg WK. Psychological implications in temporomandibular pain and dysfunction. Oral Sci Rev 1976;7:3-30.

15. I reland VE. The problem of the clicking jaw. J Prosthet Dent 1953:3:200-12.

16. Ohnishi M. Clinical application of arthroscopy in the temporomandibular joint diseases. Bull Tokyo M ed D ent U niv 1980;27:141-50. 
17. Sanders B. A rthroscopic surgery of the temporomandibular joint: Treatment of internal derangement with persistent closed lock. Oral Surg Oral Med Oral Pathol 1986;62:361-72.

18. Nitzan DW, Dolwick M F, M artinez GA. Temporomandibular joint arthrocentesis: A simplified treatment for severe, limited mouth opening. J Oral M axillofac Surg 1991;49:1163-67.

19. Sicher H. Temporomandibular articulation in mandibular overclosure. J A m D ent A ssoc 1948;36:131-39.

20. Rees $L A$. The structure and function of the temporomandibular joint. BrDent J 1954;96:125-33.

21. Rasmussen OC. Description of population and progress of symptoms in a longitudinal study of temporomandibular arthropathy. Scand J Dent Res 1981;89:196-203.
22. Kononen $M$, Waltimo $A$, Nystrom $M$. Does clicking in adolescence lead to painful temporomandibular joint locking? L ancet 1996;347:1080-81.

23. Solberg WK, W oo M W , Houston JB. Prevalence of mandibular dysfunction in young adults. J A m Dent A ssoc 1979;98:25-34.

24. Clark GT, Seligman DA, Solberg W K, et al. Guidelines for the examination and diagnosis of temporomandibular disorders. J Craniomandib Disord 1989;3:7-14.

25. Ohrbach R, Gale EN. Pressure pain thresholds, clinical assessment and differential diagnosis: Reliability and validity in patients with myogenic pain. Pain 1989;39:157-69.

26. J ohnstone $D R$, Templeton $M$. The feasibility of palpating the lateral pterygoid muscle. J Prosthet Dent 1980;44:318-23. 